\title{
Could stoma reduce the risk of rectovaginal fistula in women with excision of deep endometriosis requiring concomitant vaginal and rectal sutures? A 363-patient comparative study
}

\author{
Horace Roman ${ }^{1}$, Valerie Bridoux ${ }^{2}$, Benjamin Merlot $^{1}$, Myriam Noailles $^{1}$, Eric Magne ${ }^{1}$, \\ Benoit Resch ${ }^{3}$, Damien Forestier ${ }^{1}$, and Jean-Jacques Tuech ${ }^{4}$ \\ ${ }^{1}$ Clinique Tivoli-Ducos \\ ${ }^{2}$ Centre Hospitalier Universitaire de Rouen \\ ${ }^{3}$ Clinique Mathilde \\ ${ }^{4}$ University Hospital, Rouen
}

July 1, 2020

\begin{abstract}
Background: Even though preventive stoma is unlikely to ensure primary healing in women with juxtaposed rectal and vaginal sutures, it may be considered, in selected patients at risk of rectovaginal fistula, to reduce fistula related complications. Objective: To assess whether a generalized use of preventive stoma reduces the rate of rectovaginal fistula in women with excision of deep endometriosis requiring concomitant vaginal and rectal sutures. Study Design: Retrospective comparative study including 363 patients with deep endometriosis infiltrating the rectum and the vagina. They were managed by either rectal disk excision or colorectal resection, concomitantly with vaginal excision, in two centers (Rouen and Bordeaux) each following differing policies concerning the use of stoma. The prevalence of rectovaginal fistula was assessed, and risk factors analysed. Results: 241 and 122 women received surgery in respectively Rouen and Bordeaux. The rate of preventive stoma was $71.4 \%$ in Rouen $(\mathrm{N}=172)$ and $30.3 \%$ in Bordeaux $(\mathrm{N}=37)$. Rectovaginal fistula were recorded in 31 cases $(8.5 \%)$ : 19 women in Rouen and 12 women in Bordeaux. Performing rectal sutures less than $8 \mathrm{~cm}$ above the anal verge increased the risk of rectovaginal fistula more than 3-fold, independently of other risk factors (OR 3.4, 95\%CI 1.3-9.1). Conclusions: No statistically significant differences were found in terms of risk of rectovaginal fistula between women with rectovaginal endometriosis managed respectively by a generalized or restrictive use of preventive stoma. A higher risk of rectovaginal fistula independently related to a low rectal stapled line, less than $8 \mathrm{~cm}$ above the anal verge.
\end{abstract}

\section{Introduction}

Rectovaginal fistula is a major complication following deep endometriosis surgery, particularly when it requires disc excision or segmental resection of the rectum along with excision of adjacent vagina ${ }^{1}$. When compared to bowel leakage, rectovaginal fistula appears more difficult to repair and may require several additional surgical procedures and a longer stools deviation time using a stoma ${ }^{1,2}$. Reducing the risk of rectovaginal fistula can be achieved by various means such as avoiding vaginal opening, placement of omentum between vaginal and rectal sutures, stitches fixing vagina to rectum and separating the two sutures, or the routine use of a transitory diverting stoma.

Guidelines for management of endometriosis specifically recommend that "clinicians refer women with suspected or diagnosed deep endometriosis management to a centre of expertise that offers all available treatments in a multidisciplinary context " 3 . More specifically, surgical management of deep endometriosis infiltrating the colon and the rectum requires multidisciplinary teams that include colorectal surgeons. Worldwide sur- 
gical steps involving removal of endometriosis nodules infiltrating the digestive tract are performed by or in collaboration with general surgeons, who bring their experience and convictions. In the literature, results of several randomised trials concerning rectal cancer surgery lend support to routine use of stoma in the prevention of postoperative rectal fistula following removal of rectal cancers ${ }^{4-6}$. However, an automatic extrapolation of their conclusions to endometriosis surgery may be ill-advised due to the considerable differences between patients managed for rectal cancer and deep endometriosis. Theoretically some of these differences may protect against postoperative rectal fistula following endometriosis surgery (patients are young women, free of preoperative radiotherapy, in good health), while others may not (e.g concomitant excision of the vagina). As juxtaposition of bowel and vaginal sutures is considered a strong risk factor for rectovaginal fistula formation, a diverting stoma is routinely used by some teams ${ }^{7,8}$ in accordance with recommendations by various working groups ${ }^{9}$. These indicate that while a stoma is unlikely to ensure primary healing, it reduces the risk of fistula related complications, such as fecal peritonitis.

The benefits of stoma in deep endometriosis surgery remain however questionable, due to the lack of comparative studies in women managed for rectal endometriosis ${ }^{10}$. Colleagues who do not favor performing preventive stoma assert that stoma systematically requires a second surgical intervention to restore the digestive tract, leading to possible aesthetic harm, residual pain, incisional hernias or subcutaneous infections or stenosis of the colorectal anastomosis ${ }^{11}$, and complications requiring secondary surgery in $8.6 \%$ of cases $^{12}$.

Our multidisciplinary team's approach to surgical management of deep rectovaginal endometriosis has been modified. In the first period from 2005 to June 2018, for women requiring concomitant excision of rectum and vagina resulting in juxtaposition of the rectal stapled line and of posterior vagina suture, we advocated maximum prevention of rectovaginal fistula by large employ of preventive stoma ${ }^{13}$. This was applied by one of the authors' (H.R.) at the Expert Center of Diagnosis and Multidisciplinary Management of Endometriosis, Rouen University Hospital until subsequent publication of French Guidelines for the Management of Endometriosis raised questions about scientific support for use of preventive stoma in endometriosis surgery ${ }^{10}$. From September 2018 onwards, the recommended approach favored a more restrictive use of stoma, reserved for stapled lines on low rectum, large vaginal excisions and other risk factors such as obesity, unsatisfactory rectal stapled line air test, presumed tension on stapled line, etc. This approach was used by the author during the second period at the Clinic Tivoli-Ducos Endometriosis Centre in Bordeaux. These circumstances resulted in two populations of women managed for similar endometriosis lesions, by comparable surgery but differing preventive stoma policy.

The aim of our study was to assess rectovaginal fistula prevalence, depending on the policy for preventive stoma use, in women managed for rectovaginal endometriosis and involving juxtaposition of rectal and vaginal sutures.

\section{Patients and methods}

Patients included in this series were managed at Rouen University Hospital, France, from February 2009 to June 2018, and at the Clinic Tivoli-Ducos in Bordeaux, France, from September 2018 to February 2020. They were prospectively enrolled in the CIRENDO database, which is financed by the G4 Group (The University Hospitals of Rouen, Lille, Amiens and Caen) and coordinated by one of the authors (H.R.). Inclusion criteria were: i) women managed for rectovaginal endometriosis infiltrating both the rectum and the vagina; ii) vaginal excision followed by posterior vaginal suture, with or without hysterectomy; iii) rectal nodule removal requiring rectal lumen opening, by either disc excision or segmental resection, followed by rectal suture or colorectal anastomosis juxtaposing the vaginal suture; iv) accurate recording of the height of the rectal suture, measured in $\mathrm{cm}$ above the anal verge. Exclusion criteria were: i) rectal nodule removal by shaving without opening of rectal lumen; ii) non-French and non-English speaking patients unable to answer the questionnaire required for inclusion in the database.

All patients were preoperatively examined by experienced gynaecological surgeons (H.R., B.R. and B.M.), who identified vaginal infiltration during clinical examination. Preoperative assessment was performed by radiologists with considerable experience in deep endometriosis and included pelvic MRI, endorec- 
tal/transvaginal ultrasound and when required, computed tomography based virtual colonoscopy. This allowed assessment of rectal nodule characteristics and identification of associated localisations involving USL, ovaries, fallopian tubes, diaphragm, urinary tract, pelvic nerves etc.

To remove rectal nodules, disc excision or colorectal segmental resection were proposed. Disc excisions were carried out using a combined laparoscopic-transanal approach, by employing either a circular stapler (a technique used by numerous teams worldwide) or a semi-circular stapler (the Rouen technique, currently used by a small number of teams in Europe) ${ }^{14}$. For multiple bowel nodules, the afore-mentioned techniques may be associated with sparing healthy bowel located between consecutive nodules ${ }^{15}$. The choice of surgical approach was made preoperatively, and patients were fully informed of the aims, risks and expected benefits of our approach. Patients then had a preoperative visit with a colorectal surgeon, and were informed of the possibility of performing diverting stoma at the end of the procedure, specifically when vaginal and rectal sutures were juxtaposed, and to reduce the risk of complications related to rectovaginal fistula.

Surgical procedure on the bowel involved one gynaecological surgeon (H.R., B.M or B.R.), one experienced colorectal surgeon, and surgeons in training. The gynaecological surgeon removed all endometriosis localisations, including the vaginal infiltration and the colorectal surgeon performed rectal suture using transanal staplers. Omental flap was systematically placed between rectal and vaginal repair sutures in patients managed in Rouen prior to 2018, but not in Bordeaux from 2018 to 2019. The decision to create a primary stoma by ileostomy or colostomy was made by both surgeons and based on intraoperative findings, such as the close proximity of vaginal and rectal sutures following vaginal and rectal excision, unsatisfactory colorectal anastomosis bubble test results, appearance of tension on the rectal stapled line, excessive intraoperative bleeding, patient obesity, etc ${ }^{12}$. However, the decision to not perform stoma was more frequent in patients managed in Bordeaux from September 2020 onwards and has since become routine in accordance with French guidelines for the management of endometriosis which highlight the lack of evidence in support of preventive stoma and omentoplasty ${ }^{10}$. Consequently, the use of both stoma and omental flap has progressively become limited to the management of rectovaginal fistulae. With the exception of the use of stoma and omentoplasty, all other surgical procedures were similar between the two centres, one author (H.R.) having practiced in Rouen until June 2018and in Bordeaux from September 2018.

Postoperative hospitalisation varied from 4 to 6 days. Clinical symptoms and body temperature were recorded 3 times/day, and assessment of blood values of C-reactive protein (CRP) and white blood cells (WBC) was routinely performed at day 4,5 and $6^{16}$. When patients presented intrarectal temperature $>38.2^{\circ} \mathrm{C}$, or a progressive increase in either CRP or WBC for two consecutive days, emergency clinical examination and computed tomography with barium enema were performed to rule out rectovaginal fistula, pelvic abscess or infected pelvic hematoma. Patients with rectovaginal fistula and without primary diverting stoma underwent emergency secondary surgery with confection of diverting stoma. In patients with hematoma or abscess but without obvious rectovaginal fistula, emergency laparoscopy was performed to drain the liquid, followed by a rectal bubble air test. Where test results were abnormal or equivocal, a secondary stoma was created prophylactically ${ }^{1,12}$.

All patients managed in Rouen and Bordeaux agreed to the prospective recording of data concerning antecedents, clinical symptoms, findings of clinical and imagery examinations, surgical procedures and postoperative outcomes through the CIRENDO (North-West Inter Regional Female Cohort for Patients with Endometriosis) database (NCT02294825). Information was obtained using self-questionnaires, surgical and histological records, while data recording, contact and follow-up were carried out by 2 clinical research technicians. Standardised gastrointestinal questionnaires were routinely used to assess pre- and post-operative digestive function: the Gastrointestinal Quality of Life Index (GIQLI) ${ }^{17}$, the Knowles-Eccersley-Scott-Symptom Questionnaire (KESS) ${ }^{18}$ and the WEXNER scale ${ }^{19}$, the Urinary Symptom Profile (USP) ${ }^{20}$. Prospective recording of data was approved by the French authority CCTIRS (Advisory Committee on information processing in healthcare research).

Statistical analysis was performed using Stata 11.0 software (StatCorp). Patient characteristics, surgical procedures, postoperative outcomes and score values were presented as numbers and percentages (qualitative 
variables) or mean and SD (continuous variables). Women managed in Rouen and Bordeaux were compared using either the Kruskal-Wallis test (continuous variables) or the Fischer exact test (qualitative variables). A logistic regression model was used to identify factors independently related to the risk of rectovaginal fistula. A P value of $<.05$ was considered statistically significant. The study was approved by the Rouen University Hospital Institutional Ethics Committee for Non-Interventional Research (E2020-53, June 30, 2020).

\section{Results}

From October 2009 to March 2020, 363 patients met inclusion criteria and were enrolled in the study. Among them, 241 and 122 women received surgery in Rouen and Bordeaux respectively. 312 women (86\% of the total sample) were managed by one gynaecological surgeon (H.R.): 223 women in Rouen (92.5\% of patients having surgery in Rouen) and 89 in Bordeaux (73\% of those managed in Bordeaux). The rate of preventive stoma was $71.4 \%$ in Rouen $(\mathrm{N}=172)$ and $30.3 \%$ in Bordeaux $(\mathrm{N}=37)$. Rectovaginal fistula was recorded in 31 cases (8.5\%): in 19 women managed in Rouen (7.9\% of women having surgery in Rouen) and in 12 women undergoing surgery in Bordeaux (9.8\% of those managed in Bordeaux).

Table 1 presents patient characteristics, the majority of which were comparable between the two groups. Patients managed in Bordeaux were older and had more frequent past history of open abdominal or pelvic surgery. Table 2 presents intraoperative findings, surgical procedures and main postoperative complications. Patients managed in Bordeaux had shorter operative time, larger rectal nodules, deep nodules more often involving both uterosacral ligaments and rectovaginal space, and more frequently underwent hysterectomy. Other surgical procedures and postoperative complications were comparable between the two groups, with the exception of bladder excision which was more frequent in patients managed in Rouen.

Table 3 presents the two groups of women stratified on performing or not performing stoma. The four groups compared were statistically different regarding rectal nodule size and vaginal infiltration (larger in the groups receiving stoma in both centres). The height of rectal stapled line was significantly lower in women undergoing stoma, particularly in those managed in Bordeaux. Women undergoing nodule excision involving sacral roots and the sciatic nerve were more likely to have a stoma. Conversely, the rectovaginal fistula rate was comparable between women managed in the two centres, with or without stoma. Rectovaginal fistula was recorded in respectively $9.4 \%, 10.8 \%, 10.1 \%$ and $7 \%$ in women managed without $(8 / 85)$ and with stoma $(4 / 37)$ in Bordeaux and in those managed without (7/69) and with stoma (12/172) in Rouen.

Table 4 presents the independent relationship between several risk factors and the likelihood of rectovaginal fistula. Performing rectal sutures at a height inferior to $8 \mathrm{~cm}$ above the anal verge led to a more than 3-fold increase in risk of rectovaginal fistula, independently of stoma confection, surgical procedure carried out on the rectum, extent of vaginal infiltration or associated excision of deep endometriosis involving pelvic nerves. When compared to women managed in Bordeaux without fistula, there was a tendency toward a lower probability of rectovaginal fistula in women receiving stoma in Rouen, however the $\mathrm{P}$ value did not reach statistical significance $(\mathrm{P}=0.075)$.

\section{Discussion}

Our study compared the risk of rectovaginal fistula in two groups of patients managed for rectovaginal endometriosis with differing policies concerning preventive stoma. Despite the stoma rate being more than halved in one group, no significant difference in risk of rectovaginal fistula following concomitant excision of rectum and vagina was found. Our study however showed that a rectal stapled line at a height of $<8 \mathrm{~cm}$ was a major risk factor for fistula. This information is useful for colleagues involved in management of severe forms of endometriosis.

The major limit of our study relates to the lack of randomisation, resulting in the presence of women with and without stoma in each group. In a randomised trial, the assignment of a patient to the arm with or without stoma is based on a unique randomisation list drawn up by a statistician, resulting in two similar groups of women to compare. In our study, as in other non-randomised series ${ }^{7,21}$, performing stoma is based 
on colorectal surgeon decision and intraoperative events. The particularity of our study relates to centrebased policies, i.e. an overall intention to carry out a stoma in patients with concomitant rectal and vaginal repair in Rouen, although no stoma was ultimately performed in a third of cases, versus an overall intention to avoid performing a stoma in similar patients in Bordeaux, where a stoma was performed in one third of the cases. These differing approaches to the use of stoma led to a comparative study, the results of which may help in planning a future randomised trial.

The second limit relates to the heterogeneity of techniques used to remove rectal nodules. Our study is observational and employs data prospectively recorded in a cohort of patients managed for endometriosis in two tertiary referral centres. As deep endometriosis is a complex disease, there is a large variation in length, width, depth and height of rectal infiltration, requiring an individual surgical approach using either full thickness disc excision or colorectal resection ${ }^{22}$. However, both approaches resulted in the presence of a rectal stapled line, juxtaposed with vaginal repair. This condition significantly increases the risk of rectovaginal fistula, independently of the technique used to remove the nodule.

The third limit relates to the "before and after" design of our study. Patients were managed in Rouen prior to those in Bordeaux, thus it is likely that the surgeon practicing in both centres benefited from more experience during the second time period. This hypothesis is supported by shorter operative times recorded in Bordeaux.

Our study presents several strengths. The comparability of the two groups is ensured by the presence of one gyneaecological surgeon in both centres, who was in charge of management of the majority of patients. Surgeon recruitment and technique did not vary between the two centres with colorectal surgeons in Bordeaux and Rouen employing the same techniques to remove the rectum, i.e. disc excision and segmental resection, despite their differing approach concerning the use of preventive stoma. All surgeons involved in this study had extensive experience in endometriosis, which would logically favour good postoperative outcomes. Patients were prospectively enrolled in a cohort and benefited from rigorous follow-up and detailed recording of pre-, intra- and post-operative data. Data were managed by dedicated research technicians, avoiding patient lost to follow up and lending support to the accuracy of the data and the validity of our results.

Our study showed that the prevalence of rectovaginal fistula was comparable between the four groups stratified on the centre, and use or not use of stoma. When compared to women managed without stoma in Bordeaux, the centre with a policy favouring a restrictive use of stoma, women receiving stoma in Rouen , a centre with large use of stoma, were found to have a tendency towards a lower rectovaginal fistula rate $(\mathrm{P}=0.075)$, after adjustment for rectal stapled line suture, surgical procedure on the rectum, size of vaginal infiltration and management of pelvic nerves (the latter item being an indicator for more complex surgery). Even though a significant difference might be revealed by a larger cohort study, expected benefits should be weighed against disadvantages related to routine stoma use. We previously showed that women with deep endometriosis benefiting from preventive stoma, had a $8.6 \%$ risk of undergoing further surgery to manage specific stoma-related complications such as bowel occlusions, haemoperitoneum or leakage of bowel suture at the stoma opening, repair of incisional hernia or stoma prolapse ${ }^{12}$. Furthermore temporary stoma has been shown to increase the risk of colorectal anastomosis stenosis in women undergoing segmental resection for endometriosis of the rectosigmoid ${ }^{11}$. All patients with stoma are required to undergo additional surgery to close the stoma and restore the digestive tract, and may engender further stoma-related disadvantages such as aesthetic harm, residual pain, stoma prolapse, incisional hernias or subcutaneous infections. The benefits from a presumed decrease in risk of rectovaginal fistula should be weighed against the above-mentioned unfavourable consequences.

The occurrence of rectovaginal fistula is a major unfavourable outcome which impacts patient postoperative well-being. Though the immediate consequences are not usually life-threatening, as stools are evacuated through the vagina and not inside the pelvis and abdomen, rectovaginal fistula repair may be challenging. Simple deviation of stools through a stoma only incidentally allows rectovaginal fistula repair, as the presence of vaginal opening in contact with a discontinuous rectal stapled line may be an obstacle to natural healing ${ }^{1}$. Rectovaginal fistula repair requires several additional procedures in almost half the patients, while stoma 
closure cannot be carried out before 10 months on average ${ }^{1}$. These additional procedures, such as secondary segmental resection or delayed colo-anal anastomosis ${ }^{1,2}$, are much more complex than the repair of incisional hernia of a stoma scar or endoscopic dilatation of colorectal anastomosis stenosis. These reasons lend support to a reduction in risk of rectovaginal fistula by performing preventive stoma, as has been demonstrated in low rectal resection for rectal cancer ${ }^{4-6}$.

It should be noted that $30.3 \%$ of patients managed in Bordeaux ultimately received a preventive stoma, colorectal surgeons estimating the risk of rectovaginal fistula to be too high. In Rouen, $28.6 \%$ of patients received no stoma, due to an estimated low risk of rectovaginal fistula. It results that an average of $40 \%$ of patients who had a stoma in Rouen were unlikely to have had a stoma performed in Bordeaux. Similarly $40 \%$ of those managed without stoma in Bordeaux would have probably had a stoma performed in Rouen. As patient characteristics and surgical procedures were comparable between the two centres, any difference in prevalence of rectovaginal fistula between the two groups is likely to stem exclusively from comparison between these $40 \%$ of patients, for whom the decision to perform a stoma or not, differed according to the centre where they were managed. In our series, this involved 50 patients in Bordeaux and 96 patients in Rouen and though these patient numbers do not provide sufficient statistical power to demonstrate a statistically significant difference, our data give an indication of expected rectovaginal fistula rates in the presence or absence of stoma. More specifically, the hypothesis of $7 \%$ rectovaginal fistula in women with large indications for stoma appears reasonable, being similar to that observed in women receiving stoma in Rouen, and to that reported in another series with a $96 \%$ stoma rate $^{8}$. A higher rate of rectovaginal fistula in women with stoma in Bordeaux (10.8\%) is also logical, as the risk of fistula was considered high enough to perform a stoma, by surgeons who would not routinely perform them. In a future randomised trial, the expected rate of rectovaginal fistula in women allocated to the arm without stoma, should be higher than $10.1 \%$ (the fistula rate in women in Rouen with no stoma). A randomisation process would allocate patients with high risk of rectovaginal fistula to the control group (30.3\% of patients who had a stoma in Bordeaux), logically resulting in fistula rates higher than those recorded in our series. An important question concerns whether or not the fistula rate in women without stoma could exceed $16 \%$, thereby surpassing the cumulative rates of rectovaginal fistula in women with stoma $(7 \%)$ and of the surgical procedures required following complications incurred from routine use of stoma $(8.6 \%)^{12}$. To our knowledge, to date, no other data in the literature provides answers to this question, highlighting the need for a randomised trial to compare rectovaginal fistula in women managed for deep rectovaginal endometriosis with or without stoma.

With regards to whether the rate of fistula in our series is valid and consistent with that observed in series published by other authors, only a few studies in the literature have focused on series of women managed for low rectal endometriosis and concomitant colpectomy, in which risk of rectovaginal fistula could be comparable to ours. Firstly in a series of 100 women undergoing low colorectal resection for deep rectovaginal endometriosis by open route, Dousset et al reported concomitant vaginal infiltration in $64 \%$ of cases, a $96 \%$ stoma rate and $6 \%$ anastomotic leakage (8). Secondly in a series of 44 women undergoing low rectal resection and colpectomy, Belghiti et al recorded a rectovaginal fistula rate of $15 \%$ in women with preventive stoma and $27 \%$ in women without stoma ${ }^{7}$. On the basis of this, our fistula rate observed in 363 patients undergoing colpectomy and excision of rectal endometriosis by either disc excision or segmental resection, appears reasonable.

In conclusion, this study did not reveal statistically significant differences in terms of risk of rectovaginal fistula between women with rectovaginal endometriosis managed respectively by a generalised or restrictive use of preventive stoma. However, our data underlines the higher risk of rectovaginal fistula independently related to a low rectal stapled line, specifically up to $8 \mathrm{~cm}$ above the anal verge. Our results also suggest that a policy of restrictive use of stoma in up to $30 \%$ of women with juxtaposed rectal and vaginal sutures does not significantly increase rectovaginal fistula risk when compared to a much larger employ of preventive stoma. A further randomised trial would enable practitioners to better weigh up the benefits in terms of rectovaginal fistula and related complications versus the risk of stoma-related complications.

Study funding/competing interest(s): The North-West Inter Regional Female Cohort for Patients with 
Endometriosis (CIRENDO) is financed by the G4 Group (The University Hospitals of Rouen, Lille, Amiens and Caen) and ROUENDOMETRIOSE Association. No financial support was received for this study. The authors declare no competing interests related to this study.

Acknowledgements: The authors are grateful to Amélie Bréant and Sophie Marinette for the management of data and Helene Braund for her help in editing the manuscript.

\section{Authors' roles:}

Horace Roman designed the trial, performed the surgery, and wrote the first draft of the manuscript. Benjamin Merlot, Benoit Resch, Damien Forestier, Valérie Bridoux, Myriam Noailles, Eric Magne and Jean-Jacques Tuech performed the surgery. Horace Roman performed data collection. All the authors contributed to the final manuscript.

\section{Details of ethical approval:}

All patients signed an informed consent before enrollement in CIRENDO database and the study was approved by the Committé d'Ethique de la Recherche Non Interventionnelle, Rouen University Hospital.

\section{Tables and figures:}

Table 1. Patient characteristics.

Table 2. Intraoperative findings, surgical procedures and main immediate complications.

Table 3. Intraoperative findings and rectovaginal fistula prevalence stratified on center and use of preventive stoma.

Table 4. Independent factors related to the probability of rectovaginal fistula (logistic regression model).

\section{References:}

1. Roman H, Bridoux V, Merlot B, Resch B, Chati R, Coget J, Forestier D, Tuech JJ. Risk of bowel fistula following surgical management of deep endometriosis of the rectosigmoid: a series of 1,102 cases. Hum Reprod, 2020, In press.

2. Corte H, Maggiori L, Treton X, Lefevre JH, Ferron M, Panis Y. Rectovaginal Fistula: What Is the Optimal Strategy?: An Analysis of 79 Patients Undergoing 286 Procedures. Ann Surg. 2015;262:855-60.

3. Dunselman GA, Vermeulen N, Becker C, Calhaz-Jorge C, D'Hooghe T, De Bie B, Heikinheimo O, Horne AW, Kiesel L, Nap A, Prentice A, Saridogan E, Soriano D, Nelen W; European Society of Human Reproduction and Embryology. ESHRE guidelines: management of women with endometriosis. Hum Reprod. 2014;29:400-12.

4. Matthiessen P, Hallböök O, Rutegård J, Simert G, Sjödahl R. Defunctioning stoma reduces symptomatic anastomotic leakage after low anterior resection of the rectum for cancer: a randomized multicenter trial. Ann Surg 2007; 246: 207-214

5. Chude GG, Rayate NV, Patris V, Koshariya M, Jagad R, Kawamoto J, et al. Defunctioning loop ileostomy with low anterior resection for distal rectal cancer: should we make an ileostomy as a routine procedure? A prospective randomized study. Hepatogastroenterology 2008;55:1562-7.

6. Shiomi A, Ito M, Maeda K, Kinugasa Y, Ota M, Yamaue H, Shiozawa M, Horie H, Kuriu Y, Saito N. Effects of a diverting stoma on symptomatic anastomotic leakage after low anterior resection for rectal cancer: a propensity score matching analysis of 1,014 consecutive patients. J Am Coll Surg 2015; 220: 186-194

7. Belghiti J, Ballester M, Zilberman S, Thomin A, Zacharopoulou C, Bazot M, Thomassin-Naggara I, Daraï E. Role of protective defunctioning stoma in colorectal resection for endometriosis. J Minim Invasive Gynecol. 2014;21:472-9. 
8. Dousset B, Leconte M, Borghese B, et al. Complete surgery for low rectal endometriosis. Long-term results of a 100-case prospective study. Ann Surg 2010; 251: 887-95.

9. Working group of ESGE, ESHRE and WES. Recommendations for the surgical treatment of endometriosis Part 2: deep endometriosis. Facts Views Vis Obgyn. 2020;11:269-297.

10. Loriau J, Petit E, Mephon A, Angliviel B, Sauvanet E. Evidence-based ways of colorectal anastomotic complications prevention in the setting of digestive deep endometriosis resection: CNGOF-HAS Endometriosis Guidelines. Gynecol Obstet Fertil Senol. 2018;46:296-300.

11. Braund S, Hennetier C, Klapczynski C, Scatarelli A, Coget J, Bridoux V, Tuech JJ. Risk of postoperative stenosis following segmental resection vs. disc excision for deep endometriosis infiltrating the rectosigmoid: a retrospective study. J Minim Invasive Gynecol 2020; In press.

12. Bonin E, Bridoux V, Chati R, Kermiche S, Coget J, Tuech JJ, Roman H. Diverting stoma-related complications following colorectal endometriosis surgery: a 163-patient cohort. Eur J Obstet Gynecol Reprod Biol. 2019;232:46-53.

13. Roman H, Bubenheim M, Huet E, Bridoux V, Zacharopoulou C, Daraï E, Collinet P, Tuech JJ. Conservative surgery versus colorectal resection in deep endometriosis infiltrating the rectum: a randomized trial. Hum Reprod. 2018;33:47-57.

14. Roman H, Darwish B, Bridoux V, Chati R, Kermiche S, Coget J et Al. Functional outcomes after disc excision in deep endometriosis of the rectum using transanal staplers: a series of 111 consecutive patients. Fertil Steril. 2017; 107:977-986

15. Millochau JC, Stochino-Loi E, Darwish B, Abo C, Coget J, Chati R, Tuech JJ, Roman H. Multiple nodule removal by disc excision and segmental resection in multifocal colorectal endometriosis. J Minim Invasive Gynecol 2018;2:139-146.

16. Scattarelli A, Carriou M, Boulet L, Chati R, Coget J, Bridoux V, Tuech JJ, Roman H. C-reactive protein assessment to predict early septic complications after laparoscopic bowel resection for endometriosis: a diagnostic study. BJOG. 2019;126:1176-1182.

17. Nieveen van Dijkum EJM, Terwee CB, Oosterveld P, van der Meulen JHP, Gouma DJ, de Haes JCJM. Validation of the gastrointestinal quality of life index for patients with potentially operable periampullary carcinoma. Br J Surg 2000; 87: 110-5.

18. Knowles CH, Eccersley AJ, Scott SM, Walker SM, Reeves B, Lunniss PJ. Linear discriminant analysis of symptoms in patients with chronic constipation. Validation of a new scoring system (KESS). Dis Colon Rectum 2000; 43: 1419-26.

19. Jorge JM, Wexner SD. Etiology and management of fecal incontinence. Dis Colon Rectum1993; 36: 77-97.

20. Haab F, Richard F, Amarenco G, et al. Comprehensive evaluation of bladder and urethral dysfunction symptoms: development and psychometric validation of the Urinary Symptom Profile (USP) questionnaire. Urology 2008;71:646-56.

21. Ledu N, Rubod C, Piessen G, Roman H, Collinet P. Management of deep infiltrating endometriosis of the rectum: Is a systematic temporary stoma relevant? J Gynecol Obstet Hum Reprod. 2018;47:1-7.

22. Donnez O., Roman H. Choosing the right surgical technique for deep endometriosis: shaving, disc excision, or bowel resection? Fertil. Steril. 2017; 108: 931-942.

\section{Hosted file}

Table 1hb.docx available at https://authorea.com/users/316235/articles/465021-could-stomareduce-the-risk-of-rectovaginal-fistula-in-women-with-excision-of-deep-endometriosisrequiring-concomitant-vaginal-and-rectal-sutures-a-363-patient-comparative-study 


\section{Hosted file}

Table 2.docx available at https://authorea.com/users/316235/articles/465021-could-stomareduce-the-risk-of-rectovaginal-fistula-in-women-with-excision-of-deep-endometriosisrequiring-concomitant-vaginal-and-rectal-sutures-a-363-patient-comparative-study

\section{Hosted file}

Table 3hb-2.docx available at https://authorea.com/users/316235/articles/465021-could-stomareduce-the-risk-of-rectovaginal-fistula-in-women-with-excision-of-deep-endometriosisrequiring-concomitant-vaginal-and-rectal-sutures-a-363-patient-comparative-study

\section{Hosted file}

Table 4hb.docx available at https://authorea.com/users/316235/articles/465021-could-stomareduce-the-risk-of-rectovaginal-fistula-in-women-with-excision-of-deep-endometriosisrequiring-concomitant-vaginal-and-rectal-sutures-a-363-patient-comparative-study 